\title{
A Cold Field Emission Gun Optimized for Cryo-EM Applications
}

Stan Konings ${ }^{1}$ and Maarten Bischoff ${ }^{2}$

${ }^{1}$ Thermo Fisher, Breda, Noord-Brabant, Netherlands, ${ }^{2}$ Thermo Fisher, Eindhoven, Noord-Brabant, Netherlands

A cold field emission gun (FEG) is characterized by its high brightness and the low energy spread of the electrons in the electron beam. The cold FEG can further be optimized for high brightness applications by increasing the extraction voltage. The higher brightness will improve the signal to noise ratio of applications like high-resolution scanning transmission electron microscopy (HR-STEM) and energy dispersive $\mathrm{x}$-ray spectroscopy mapping. On the other hand, some applications favor a further lowering of the energy spread which can be achieved by lowering the extraction voltage. One example is electron energy loss spectroscopy (EELS), where the lower energy spread will increase the resolution of the energy spectrum. Another example is high-resolution transmission electron microscopy (HR-TEM) where the lower energy spread leads to better contrast at high frequencies by lowering the impact of the chromatic aberration. This will lead to higher spatial resolution of the 2D image.

The flexibility to tune the microscope for high brightness or low energy spread is advantageous for microscopes used for material science applications, where there is a need to characterize the specimen with a variety of (analytical) imaging techniques. This flexibility is much less required in life science applications like cryo-EM single particle analysis (SPA) and tomography that are mostly performed in HR-TEM mode. The stability requirements are however more stringent for cryo-electron microscopes since especially SPA requires fully automated data acquisition runs which can take from several hours to several days.

To further optimize the 3D resolution of proteins and protein complexes obtained by cryoEM [1], the Thermo Fisher high-end cryoEM (Krios) can now be configured with a $300 \mathrm{kV}$ CFEG. In this presentation we will present first results obtained by this CFEG which is optimized for life science applications (ECFEG). The higher contrast due to the lower energy spread will be demonstrated (Figure 1) and it will be explained why the lower brightness at this setting is not impacting the life science application. In addition, we will show results on the excellent beam current stability. The beam current stability is directly related to the vacuum level at the tip of the FEG, which is to a large extent determined by electron stimulated desorption (ESD). Since the E-CFEG is operated at a lower total beam current ( $5 \mathrm{nA}$ ), the ESD is also lower which leads to extended beam current stability (Figure 2). After the beam current drops below a certain level, the tip must be regenerated via a short heating of the tip. This procedure is called tip flashing and takes less than a minute and must be done about every 6-10 hours. An autoflash is embedded in the SPA data acquisition program EPU and is triggered automatically during the automated data acquisition run based on the monitored level of the beam current. 

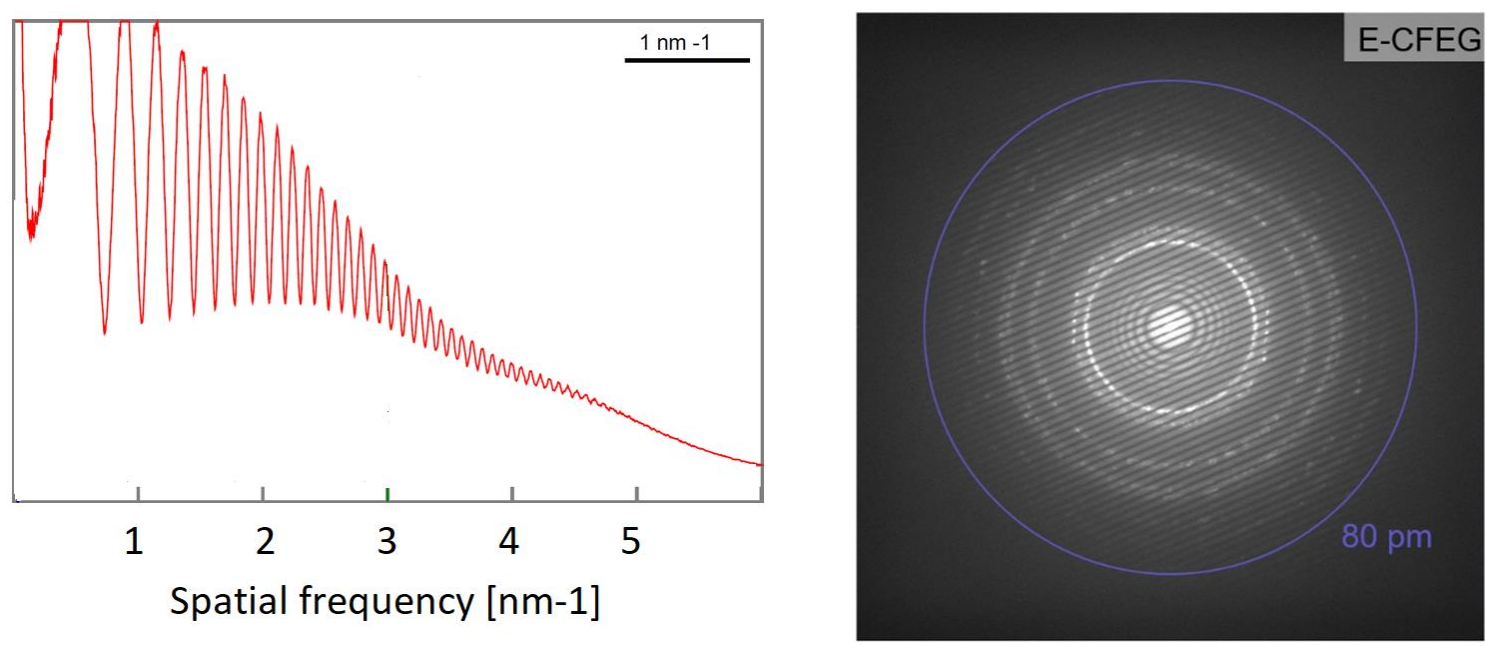

Figure 1. Thon ring image on amorphous carbon (left) and Young's fringe image obtained on a gold on amorphous carbon cross grating (right) as obtained with the E-CFEG on Thermo Fisher Krios at $300 \mathrm{kV}$

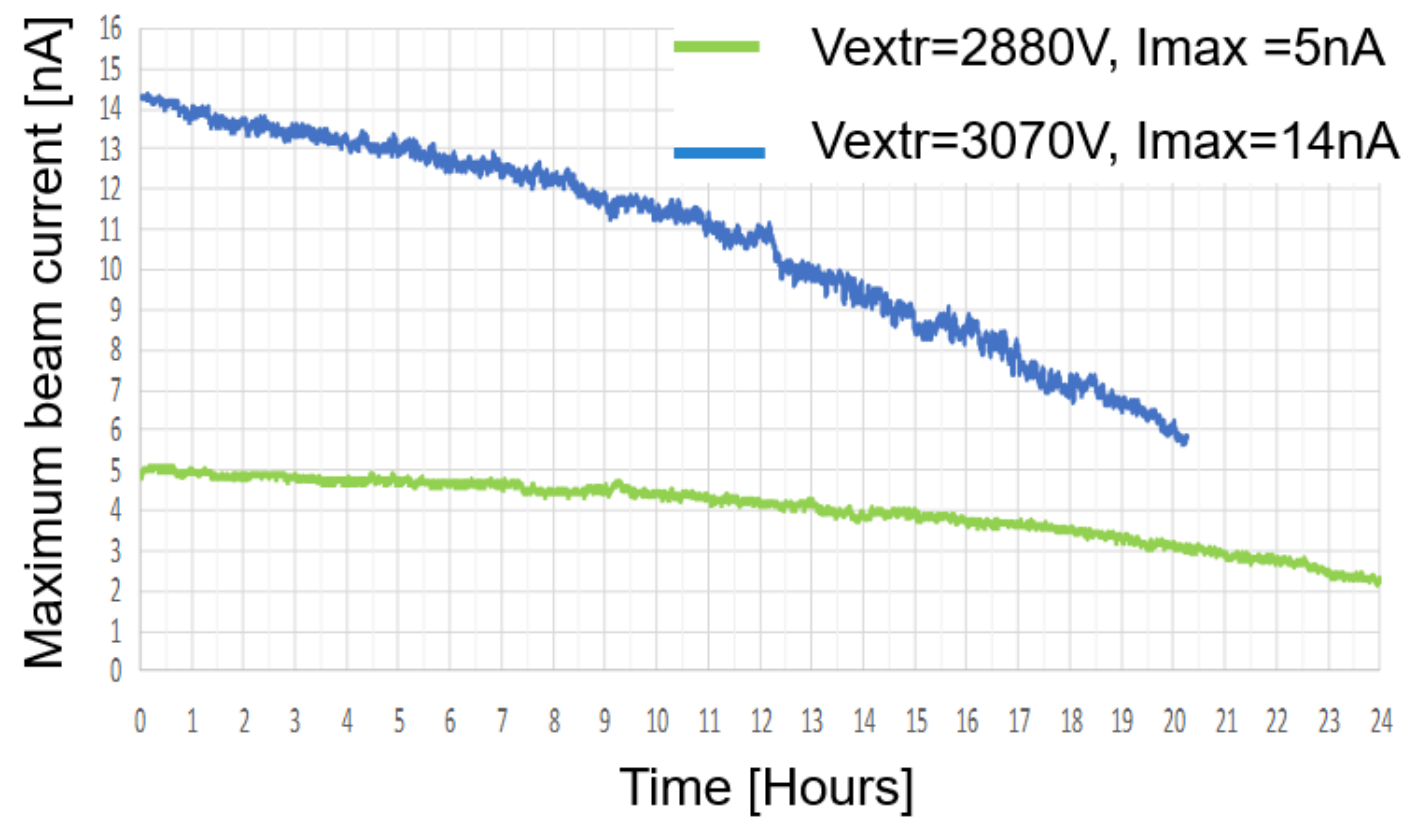

Figure 2. Cold FEG beam current stability for high brightness mode (blue) and low energy spread mode (green). The E-CFEG is operated in low energy spread mode.

References

[1] Nature Methods, Special feature: Method of the Year 2015 (2015) 